\title{
Análisis de la técnica de tiro libre de jugadores de baloncesto en contexto
}

\author{
formativo
}

\section{Analysis of the free throw technique in formative basketball}

\section{Análise da técnica do lance livre de jogadores de basquete padronizados em contexto de treinamento}

\author{
Díaz-Aroca, A., y Arias-Estero, J. L. \\ UCAM Universidad Católica San Antonio de Murcia
}

\begin{abstract}
RESUMEN
El objetivo fue determinar si la técnica de tiro libre ejecutada por jugadores de baloncesto de entre 9 y 11 años no participantes en ninguna selección autonómica y nacional coincidió con la técnica reportada por la literatura y estudios previos. El trabajo se realizó diferenciando la técnica de tiro antes de la salida y a la salida del balón. Participaron 12 jugadores varones de entre 9 y 11 años $(M=10,43, D T=, 63)$ que nunca habían participado en ninguna selección autonómica ni nacional. Se utilizó la metodología observacional a través de un diseño puntual/nomotético/multidimensional. Se grabó a los jugadores realizando un test que constaba de 10 tiros libres. Posteriormente, dos observadores observaron la ejecución técnica de cada uno de los tiros realizados por los jugadores. Se realizó la detección de t-patterns mediante el software Theme v.6 EDU. No se detectó ningún tpattern antes de la salida del balón ni a la salida debido a que los tiros se realizaron siguiendo conductas muy dispares, que no se repitieron de forma regular en el tiempo. En el presente trabajo se utilizó un instrumento de observación que permitió la obtención de información directamente aplicable a la realidad diferenciando lo que ocurre antes de la salida del balón y a la salida del mismo. Se comprobó que los jugadores analizados, los cuales no jugaban en ninguna selección, ejecutaron una técnica de tiro que contrastaba en varios criterios con las recomendaciones propuestas por la literatura y estudios previos.
\end{abstract}

Palabras clave: metodología observacional, t-patterns, minibasket, iniciación deportiva, deportes de equipo.

\begin{abstract}
The purpose was to determine whether the free throw technique of under-12 basketball players, who did not participated in any regional and national team, was similar to the technique reported by the literature and previous studies. The analysis differentiated prior to shoot and at ball release moments. Participant were 12 male players between 9 and 11 years old $(M=10.43, S D=, 63)$ who had never participated in any regional and national team. The observational methodology was used through a point/nomothetic/multidimensional design. The players were recorded performing a test consisting of 10 free throws. Two observers observed the technical execution of each of the shots made by the players. T-patterns were detected using Theme v.6 EDU software. Neither prior to ball release nor at ball release t-patterns were detected because the free throws followed very different behaviours, which were not repeated regularly over time. In the present work, an observation instrument was used that allowed obtaining information directly applicable to reality, differentiating between the free throw technique prior to shoot
\end{abstract}

Correspondence to: José Luis Arias-Estero. Dirección Postal: Campus de los Jerónimos, 137, Universidad Católica San Antonio de Murcia, Murcia (España).Email: jlae84@hotmail.com 


\section{Díaz-Aroca, \& Arias-Estero}

and at ball release moments. The analysed players, who did not play in any regional and national team, executed a free throw technique that contrasted in several criteria with the literature and previous studies recommendations.

Keywords: observational methodology, t-patterns, mini-basketball, youth sport, team sports.

\section{RESUMO}

O objetivo foi determinar se a técnica do tiro livre executada por jogadores de basquete entre 9 e 11 anos de idade que não participavam de nenhuma seleção regional e nacional coincidia com a técnica relatada na literatura e em estudos anteriores. O trabalho foi realizado diferenciando a técnica do chute antes do início e do início da bola. Participaram 12 jogadores do sexo masculino entre 9 e 11 anos $(M=10,43, D T=63)$ que nunca haviam participado de nenhuma equipe regional ou nacional. A metodologia observacional foi utilizada através de um desenho pontual/nomotético/multidimensional. Os jogadores foram gravados realizando um teste composto por 10 chutes da linha de lance livre. Posteriormente, dois observadores observaram a execução técnica de cada um dos tiros feitos pelos jogadores. Os t-patterns foram detectados usando o software Theme v.6 EDU. Nenhum t-patterns foi detectado antes do início da bola ou no início, porque os chutes foram feitos seguindo comportamentos muito diferentes, que não foram repetidos regularmente ao longo do tempo. No presente trabalho, foi utilizado um instrumento de observação que permitiu obter informações diretamente aplicáveis à realidade, diferenciando o que ocorre antes da saída da bola e da saída da bola. Verificou-se que os jogadores analisados, que não jogaram em nenhuma seleção, executaram uma técnica de chute que contrasta em vários critérios com as recomendações propostas pela literatura e estudos anteriores.

Palavras chave: metodologia observacional, T-patterns, mini-basquetebol, iniciação esportiva, esportes coletivos.

\section{INTRODUCCIÓN}

El tiro a canasta ha sido ampliamente estudiado por ser la acción técnico-táctica más determinante en baloncesto (Li y Feng, 2020; Maglott, Chiasson, y Shull, 2019). A diferencia del tiro de campo, el cual puede verse afectado por la interferencia de un oponente, el tiro libre depende únicamente del jugador que lo realiza (Tran y Silverberg, 2008). No obstante, la mayoría de los trabajos que estudian el tiro libre se enmarcan en baloncesto de alto rendimiento o con jugadores adultos (Lorenzo, Lorenzo, Conte, y Giménez, 2019; Morgulev, Azar, y Bar-Eli, 2019). Aquellos que se han preocupado por su estudio en baloncesto con niños analizaron a jugadores de alto nivel, como jugadores de selecciones autonómicas (Garzón, Lapresa, Anguera, y Arana, 2011, 2014). Sin embargo, la actual realidad del baloncesto en edad de formación contrasta con los participantes investigados hasta la fecha. Atendiendo a que la primera etapa donde los jugadores pueden participar con sus selecciones autonómicas es en la categoría alevín (minibasket, normalmente entre los 9 y 11 años), la gran mayoría nunca son seleccionados para disputar un campeonato de ese nivel. Por ejemplo, durante la temporada 2019 / 2020, de los 48 equipos alevines que componen la categoría en una federación autonómica concreta, tan solo 12 ó 14 jugadores serán seleccionados para participar en el Campeonato de España de Minibasket. Por el contrario, alrededor del $97,5 \%$ de los jugadores no participarán a dicho nivel. Es por ello que parece justificado plantear estudios sobre el tiro libre en baloncesto de menos de 12 años cuyos participantes sean representativos de la realidad general de este deporte.

El tiro libre es una acción cerrada, cuyo análisis en profundidad requiere diferenciar dos momentos determinados por el punto en el que el balón abandona las manos del jugador (De Oliveira, Oudejans, y Beek., 2006; Klostermann, Panchuk, y Farrow 2017; Okubo y Hubbard, 2016). Antes de la salida del balón, el tiro libre debe ser ejecutado sin salto, estilo alto, utilizando una mano de tiro y la otra de apoyo, pies separados aproximadamente la anchura de los hombros y apuntando hacia el aro. A la salida del balón, el brazo debe quedar orientado hacia la canasta, codo extendido, brazo próximo a la vertical, muñeca flexionada, el balón debe salir en el punto de máxima altura, sin rotación y sin desplazamiento del centro de gravedad (American Sport Education Program [ASEP], 1996; Del Rio, 2002; Krause y Nelson, 2019; Montero, 2013).

En baloncesto de menos de 12 años es reducido el número de estudios que analizaron el tiro libre. Cuatro de esos estudios demostraron los efectos 


\section{Técnica de tiro libre en baloncesto formativo}

positivos de la reducción del tamaño del balón y la altura de la canasta empleados en baloncesto adulto sobre la auto-eficacia de los participantes y la precisión, éxito y mejora de la técnica (Chase, Ewing, Lirgg, y George, 1994; Isaacs y Karpman, 1981; Regimbal, Deller, y Plimpton, 1992; Satern, Messier, y Keller-McNulty, 1989). Arias (2012a) obtuvo que un balón de menor masa que el usado en competición oficial de minibasket no aumentó la precisión y eficacia del tiro libre. Da Silva, Pereira-Monfredini, y Teixeira (2017) y Perreault y French (2015) comprobaron el efecto positivo del feedback en el aprendizaje del tiro libre. Finalmente, Garzón et al. (2011) diseñaron una herramienta observacional que permitió detectar patrones temporales (t-patterns) durante el tiro libre. Posteriormente, se empleó dicho análisis de t-patterns en el estudio del tiro libre para demostrar la no existencia de rutinas gestuales previas (Lapresa, García, Arana, y Garzón, 2011), y que el cambio reglamentario de la categoría alevín (menos de 12 años) a la infantil (menos de 14 años) provocó un empeoramiento de la técnica y una disminución del éxito (Garzón et al., 2014).

El uso de los t-patterns para analizar la técnica de tiro libre en baloncesto de menos de 12 años supuso un punto de inflexión porque permitió el descubrimiento de conductas ocultas susceptibles de entrenamiento. Brevemente, esta técnica se basa en un poderoso algoritmo que detecta estructuras ocultas en el registro, correspondiente a acciones compuestas de códigos concurrentes que ocurren en el mismo orden, con distancias temporales entre sí en cuanto a número de frames (Magnusson, 1996, 2000). Recientemente, su aplicación ha mostrado grandes avances en el análisis de la técnica en diferentes deportes, como judo (Prieto-Lage, Rodríguez-Souto, Prieto, y Gutiérrez-Santiago, 2020) o fútbol (Prieto-Lage, Artigues-Ribas, y Gutiérrez-Santiago, 2020; Diana et al., 2017).

En resumen, aunque la metodología observacional se ha posicionado como la más apropiada para analizar la técnica de tiro libre en un contexto real o habitual sin llegar a interferir (Anguera y Hernández-Mendo, 2014), los estudios revisados en baloncesto de formación evidenciaron lo siguiente. Primero, los jugadores que no forman parte de selecciones no han sido analizados, a pesar de que esta población es la más abundante. Segundo, al instrumento de observación diseñado por Lapresa et al. (2011) para que los entrenadores pudiesen analizar la técnica de tiro libre de forma sencilla, se podrían añadir otros criterios que permitan incidir sobre lo que ocurre antes y a la salida del balón. El objetivo del presente trabajo fue determinar si la técnica de tiro libre ejecutada por jugadores de baloncesto de entre 9 y 11 años no participantes en ninguna selección coincidió con la técnica reportada por la literatura y estudios previos. Para ello, el trabajo se realizó diferenciando la técnica de tiro antes de la salida y a la salida del balón. La principal hipótesis fue que la técnica de tiro sería diferente a aquella reportada por la literatura y jugadores de selecciones de estudios previos.

\section{MATERIAL Y MÉTODOS}

\section{Participantes}

Los participantes fueron 12 jugadores varones de entre 9 y 11 años $(M=10,43, D T=, 63)$ que nunca habían participado en ninguna selección autonómica ni nacional. Ellos fueron seleccionados intencionadamente debido a que tenían características homogéneas relacionadas con: (a) experiencia previa jugando a baloncesto federado, (b) nivel de fuerza, (c) altura, y (d) peso (tabla 1). Todos los jugadores pertenecían al mismo club, en la misma temporada, de una localidad situada en la Región de Murcia. Todos ellos aceptaron: (a) participar en el test programado y (b) ser grabados durante la prueba. Los padres de los participantes completaron formularios de consentimiento informado y los participantes dieron su consentimiento. El Comité de Ética en Investigación de la Universidad aprobó el estudio, que se realizó de acuerdo con la Declaración de Helsinki.

Tabla 1

Características de los participantes

\begin{tabular}{lccc}
\hline Característica & $M$ & $S D$ & $p$ \\
\hline $\begin{array}{l}\text { Experiencia } \\
\text { previa jugando a } \\
\text { baloncesto }\end{array}$ & 3.22 & .10 & $>.05$ \\
$\begin{array}{l}\text { federado (años) } \\
\text { Nivel de fuerza }\end{array}$ & 19.25 & 1.10 & $>.05$ \\
(Nw) & 1.33 & .27 & $>.05$ \\
Altura (m) & 38.6 & 8.75 & $>.05$ \\
Peso (Kg) & & & \\
\hline
\end{tabular}




\section{Díaz-Aroca, \& Arias-Estero}

\section{Diseño}

En el presente trabajo se utilizó la metodología observacional (Anguera y Hernández-Mendo, 2014). El diseño fue puntual/nomotético/multidimensional (Anguera, Blanco-Villaseñor, Hernández-Mendo, y Losada, 2011). Este diseño se ubica en el cuadrante III. Puntual, ya que no hubo seguimiento de los jugadores y los participantes fueron testados en un único día. Nomotético, porque fueron distintos participantes no analizados como una única unidad. Multidimensional, ya que se consideraron diversos niveles de respuesta dentro del instrumento de observación. Se grabó a los jugadores realizando un test que constaba de 10 tiros desde la línea de tiro libre. Posteriormente, dos observadores observaron la ejecución técnica de cada uno de los tiros realizados por los jugadores.

\section{Material}

Se utilizó un instrumento observacional compuesto por una combinación entre un sistema de categorías y un formato de campo (Garzón et al., 2014; Lapresa et al., 2011) formado por 17 criterios y 47 categorías (tabla 2). Este instrumento fue validado en un estudio previo (ver Autores). Los criterios fueron agrupados diferenciándolos antes de la salida del balón y a la salida del mismo. Para verificar la validez de constructo del instrumento, a parte de la coherencia con el marco teórico y la observación exploratoria realizada, se utilizó una escala Likert de 5 niveles. A través de la escala se preguntó a cuatro entrenadores de baloncesto su nivel de conformidad con el instrumento diseñado. Ellos contaban con una experiencia de al menos cinco años como entrenadores y habían participado en estudios previos con metodología observacional. Todos ellos coincidieron en conceder al instrumento el mayor valor de la escala. La observación de las grabaciones y su registro se realizó mediante un instrumento informatizado ad hoc formado por una hoja de Excel automatizada junto con el programa Virtual Dub. En este instrumento informático de registro se introdujeron los códigos correspondientes a cada una de las opciones de los criterios variables.

Tabla 2

Descripción de la herramienta de observación. Se han dividido los criterios antes de la salida del balón (8 primeros) y a la salida del balón (9 últimos)

\begin{tabular}{|c|c|c|c|}
\hline Criterio & Categoría & Código & Descripción \\
\hline \multicolumn{4}{|c|}{ Antes de la salida del balón } \\
\hline \multirow{3}{*}{ Tipo de Tiro } & Sin salto & SSA & El jugador no realiza un salto en el momento del tiro \\
\hline & Con salto & CSA & El jugador realiza un salto en el momento del tiro \\
\hline & Alto & ETA & $\begin{array}{l}\text { Balón por encima del nivel de los ojos antes del inicio del } \\
\text { tiro }\end{array}$ \\
\hline Estilo de Tiro & Bajo & ETB & Balón por debajo o altura de la línea de los ojos antes del tiro \\
\hline & Una mano & UMA & $\begin{array}{l}\text { Sólo una mano (derecha o izquierda) aporta el impulso al } \\
\text { balón al inicio del tiro }\end{array}$ \\
\hline \multirow{2}{*}{ Mano de Tiro } & Ambas manos & AMA & Las dos manos aportan el impulso al balón al inicio del tiro \\
\hline & Por debajo & CUD & Sólo hay una mano de tiro y esta se coloca por debajo \\
\hline Colocación Mano & Lateral & CUL & Sólo hay una mano de tiro y esta se coloca por el lateral \\
\hline \multirow[t]{2}{*}{ Tiro al Inicio } & Por debajo y lateral & $\mathrm{CDL}$ & Hay dos manos de tiro, una por debajo y otra lateral al balón \\
\hline & Ambas laterales & CAL & Hay dos manos de tiro y ambas se colocan laterales al balón \\
\hline \multirow{3}{*}{$\begin{array}{l}\text { Colocación Mano } \\
\text { Apoyo Inicio }\end{array}$} & No hay mano de apoyo & SMA & Hay dos manos de tiro y ambas se colocan laterales al balón \\
\hline & Lateral & MIL & Hay una mano de apoyo y esta se coloca en el lateral \\
\hline & Por debajo & MID & Hay una mano de apoyo y esta se coloca por debajo \\
\hline
\end{tabular}




\section{Técnica de tiro libre en baloncesto formativo}

Por encima

\section{Flexión Brazo al \\ Inicio}

Orientación de Pies

Disposición de Pies

Muy flexionado

Flexión media

Ambos pies hacia canasta

MIE

MFL

FME

DPC

SPJ

Ningún pie hacia canasta

NPO

Ambos a la misma

DPI

distancia

Pie del brazo ejecutor PEA adelantado

Pie contrario ejecutor PCE

adelantado

\section{A la salida del balón}

Momento salida

balón

Colocación mano de

apoyo a la salida del

balón

Localización mano

tiro a la salida del

balón

Orientación del

brazo

Extensión del brazo

a la salida del balón

Verticalidad del

brazo a la salida del

balón

Estado de la muñeca

a la salida del balón
Punto de máxima altura

Antes del punto máxima

$\mathrm{PMH}$ altura

\section{Estática lateral}

Se mueve hacia abajo

No hay mano de apoyo

Encima de la línea de los ojos

Debajo de la línea de los ojos

\section{A canasta}

Hacia el interior

Totalmente extendido

Poco extendido

Muy flexionado

Brazo muy próximo a la vertical

Brazo próximo a la BPR vertical

Brazo poco próximo a la BPP vertical

Flexión completa

FCO

Flexión incompleta

BMP
Hay una mano de apoyo y esta se coloca por encima

El ángulo formado entre antebrazo y brazo es < a $90^{\circ}$

El ángulo formado entre antebrazo y brazo es próximo a $90^{\circ}$

Los dos pies orientados hacia canasta

El pie correspondiente al brazo ejecutor orientado a canasta

Ningún pie orientado a canasta

Los dos pies a la misma distancia

Pies correspondiente al brazo ejecutor adelantado

Pie contrario al brazo ejecutor adelantado

En el punto de máxima altura de salto

En la fase ascendente del salto

ELA La mano se mantiene estable después de la salida del balón

HAB La mano se mueve hacia abajo después de la salida del balón

NMA Cuando las dos manos participan en dar impulso al balón

ELO La mano se encuentra por encima de la línea de los ojos

DLO Cuando la mano está por debajo o el balón obstruye la visión

HCA El brazo de tiro se queda apuntando hacia canasta

HIN El brazo de tiro se orienta hacia el interior del cuerpo

TEX Antebrazo y brazo forman un ángulo de $180^{\circ}$ o muy próximo

PEX Antebrazo y brazo forman un ángulo < a $180^{\circ}$ y > a $90^{\circ}$

MFX El ángulo entre el antebrazo y el brazo es $<90^{\circ}$ o próximo

Entre el brazo y el plano sagital existe un ángulo $<$ a $45^{\circ}$

Entre el brazo y el plano sagital hay un ángulo próximo a $45^{\circ}$

Entre el brazo y el plano sagital existe un ángulo > a $45^{\circ}$

FIC No se flexiona completamente la muñeca al tirar 


\section{Díaz-Aroca, \& Arias-Estero}

$\begin{array}{llll} & \text { Grande hacia delante } & \text { DGD } & \text { Distancia > de medio pie entre punto despegue y aterrizaje } \\ \text { Desplazamiento del } & \begin{array}{l}\text { Moderado hacia delante } \\ \text { cuerpo al tirar }\end{array} & \text { DMD } & \text { Distancia < de medio pie entre punto despegue y aterrizaje } \\ & \text { Grande hacia atrás } & \text { DGA } & \text { Distancia > de medio pie entre punto despegue y aterrizaje } \\ & \begin{array}{l}\text { Sin desplazamiento } \\ \text { Sin rotación }\end{array} & \text { SDE } & \text { No existe una distancia entre punto de despegue y aterrizaje } \\ \text { Rotación del cuerpo } & \text { Rotación hacia derecha } & \text { ROD } & \text { Existe un cambio en la rotación del cuerpo hacia la derecha } \\ & \text { Rotación hacia izquierda } & \text { ROI } & \text { Existe un cambio en la rotación del cuerpo hacia la izquierda }\end{array}$

\section{Procedimiento}

Se procedió a la filmación de los jugadores ejecutando el test de tiro. Este consistió en realizar 10 lanzamientos desde la línea de tiros libres siguiendo las reglas de juego oficiales al respecto (altura del aro 2,6 m y distancia del tiro libre $4 \mathrm{~m}$ ). Se realizó en parejas, de modo que un participante realizaba los tiros y el otro le pasaba el balón. Cuando la pareja terminaba de ejecutar el test, otra pareja lo comenzaba. El orden de participación de los participantes fue aleatorio. Se pidió a los jugadores que ejecutaran el tiro de la misma forma que lo hacían en un partido real. Esto es, una vez que el participante tenía el balón para tirar disponía de 5 segundos. Se utilizaron dos cámaras (Everio Full HD-GZ-GX1BE, JVC, Japan), a una altura de 1,30 m y a $5 \mathrm{~m}$ del jugador. Una se situó lateral y paralela al jugador en posición de tiro, para que se visualizara al jugador, la trayectoria del balón y la canasta. La otra cámara se situó debajo de la canasta de manera que se visualizara al jugador frontalmente, así como la trayectoria de salida del balón. Se formó específicamente durante 25 horas a dos observadores para la observación de los criterios concretos del presente estudio. Estos observadores contaban con al menos cinco años de experiencia como entrenadores en baloncesto de menos de 12 años y más de 300 horas de experiencia en la observación de acciones de juego en dicha categoría.

\section{Análisis de calidad del dato}

Para el análisis de Generalizabilidad se ha seguido el trabajo de Blanco-Villaseñor, Castellano, Hernández-
Mendo, Sánchez-López y Usabiaga (2014) que se fundamenta en (a) definición de las facetas de estudio, (b) análisis de varianza de las puntuaciones obtenidas sobre las facetas de estudio, (c) cálculo de los componentes de error, (d) optimización de los coeficientes de Generalizabilidad. En términos operativos, esto implicó tres aplicaciones: (a) estudio de la validez, (b) estudio de la fiabilidad, (c) estimación de la muestra. En cuanto a la validez, se consideró válido dado que fue un instrumento establecido y empleado previamente (Autores).

La fiabilidad intra- e inter-observadores al final de la formación fue calculada (coeficiente Kappa (Cohen, 1968) $>$,96, R de Pearson $=1$, Rho de Spearman $=1$, Tau-B de Kendall $=1$, porcentaje de acuerdo $>93 \%$, Tabla 3).

En total se observaron y registraron 120 tiros ejecutados por los jugadores. Ambos observadores observaron y registraron todos los tiros. Se calculó la fiabilidad de la observación mediante las estrategias intra- e inter-observador obteniendo unos valores adecuados (coeficiente Kappa $=1, \mathrm{R}$ de Pearson $=1$, Rho de Spearman $=1$, Tau-B de Kendall $=1$, porcentaje de acuerdo $=100 \%$, Tabla 4).

El informe obtenido mediante el programa SAGT (Hernández-Mendo, Blanco-Villaseñor, Pastrana, Morales-Sánchez, y Ramos-Pérez, 2016) mostró un porcentaje de varianza del $100 \%$ (Criterios:Tiros:Jugadores). El coeficiente de generalizabilidad relativo (fiabilidad) fue $1 \mathrm{y}$ el coeficiente de generalizabilidad absoluto (generalizabilidad) es 0.91 . 


\section{Técnica de tiro libre en baloncesto formativo}

Tabla 3

Fiabilidad intra- e inter-observadores al final de la formación

\begin{tabular}{|c|c|c|c|c|c|}
\hline Criterio & Kappa & $\begin{array}{c}\mathrm{R} \mathrm{de} \\
\text { Pearson }\end{array}$ & $\begin{array}{c}\text { Rho de } \\
\text { Spearman }\end{array}$ & $\begin{array}{l}\text { Tau-B de } \\
\text { Kendall }\end{array}$ & $\begin{array}{c}\% \text { de } \\
\text { acuerdo }\end{array}$ \\
\hline \multicolumn{6}{|l|}{ Antes de la salida } \\
\hline Tipo de Tiro & 1 & 1 & 1 & 1 & 100 \\
\hline Estilo de Tiro & 1 & 1 & 1 & 1 & 100 \\
\hline Mano de Tiro & 1 & 1 & 1 & 1 & 100 \\
\hline Colocación Mano Tiro al Inicio & 1 & 1 & 1 & 1 & 100 \\
\hline Colocación Mano Apoyo Inicio & 1 & 1 & 1 & 1 & 100 \\
\hline Flexión Brazo al Inicio & 1 & 1 & 1 & 1 & 100 \\
\hline Orientación de Pies & 1 & 1 & 1 & 1 & 100 \\
\hline Disposición de Pies & 1 & 1 & 1 & 1 & 100 \\
\hline \multicolumn{6}{|l|}{ A la salida } \\
\hline Momento salida balón & 1 & 1 & 1 & 1 & 100 \\
\hline Colocación mano de apoyo a la salida del balón & 1 & 1 & 1 & 1 & 100 \\
\hline Localización mano tiro a la salida del balón & 1 & 1 & 1 & 1 & 100 \\
\hline Orientación del brazo & 1 & 1 & 1 & 1 & 100 \\
\hline Extensión del brazo a la salida del balón & 1 & 1 & 1 & 1 & 100 \\
\hline Verticalidad del brazo a la salida del balón & ,96 & 1 & 1 & 1 & 93 \\
\hline Estado de la muñeca a la salida del balón & 1 & 1 & 1 & 1 & 100 \\
\hline Desplazamiento del cuerpo al tirar & 1 & 1 & 1 & 1 & 100 \\
\hline Rotación del cuerpo & 1 & 1 & 1 & 1 & 100 \\
\hline
\end{tabular}

Tabla 4

Fiabilidad intra-e inter-observadores al final de la observación

\begin{tabular}{|c|c|c|c|c|c|}
\hline Criterio & Kappa & $\begin{array}{c}\mathrm{R} \text { de } \\
\text { Pearson }\end{array}$ & $\begin{array}{c}\text { Rho de } \\
\text { Spearman }\end{array}$ & $\begin{array}{l}\text { Tau-B de } \\
\text { Kendall }\end{array}$ & $\begin{array}{c}\% \text { de } \\
\text { acuerdo }\end{array}$ \\
\hline \multicolumn{6}{|l|}{ Antes de la salida } \\
\hline Tipo de Tiro & 1 & 1 & 1 & 1 & 100 \\
\hline Estilo de Tiro & 1 & 1 & 1 & 1 & 100 \\
\hline Mano de Tiro & 1 & 1 & 1 & 1 & 100 \\
\hline Colocación Mano Tiro al Inicio & 1 & 1 & 1 & 1 & 100 \\
\hline Colocación Mano Apoyo Inicio & 1 & 1 & 1 & 1 & 100 \\
\hline Flexión Brazo al Inicio & 1 & 1 & 1 & 1 & 100 \\
\hline Orientación de Pies & 1 & 1 & 1 & 1 & 100 \\
\hline Disposición de Pies & 1 & 1 & 1 & 1 & 100 \\
\hline \multicolumn{6}{|l|}{ A la salida } \\
\hline Momento salida balón & 1 & 1 & 1 & 1 & 100 \\
\hline Colocación mano de apoyo a la salida del balón & 1 & 1 & 1 & 1 & 100 \\
\hline Localización mano tiro a la salida del balón & 1 & 1 & 1 & 1 & 100 \\
\hline Orientación del brazo & 1 & 1 & 1 & 1 & 100 \\
\hline Extensión del brazo a la salida del balón & 1 & 1 & 1 & 1 & 100 \\
\hline Verticalidad del brazo a la salida del balón & 1 & 1 & 1 & 1 & 100 \\
\hline Estado de la muñeca a la salida del balón & 1 & 1 & 1 & 1 & 100 \\
\hline Desplazamiento del cuerpo al tirar & 1 & 1 & 1 & 1 & 100 \\
\hline Rotación del cuerpo & 1 & 1 & 1 & 1 & 100 \\
\hline
\end{tabular}

\section{Análisis estadístico}

A partir del archivo de datos generado tras la observación se realizó la detección de t-patterns mediante el software Theme v.6 EDU (Magnusson,
Burgoon, y Casarrubea, 2016). El análisis se realizó sobre los criterios que componen la técnica de tiro antes de la salida del balón y a la salida del balón de cada uno de los jugadores. Se establecieron los siguientes parámetros de búsqueda. La frecuencia de 


\section{Díaz-Aroca, \& Arias-Estero}

aparición de al menos un $80 \%$ de todos los eventos tipo se situó por debajo de la ocurrencia seleccionada. En todo caso nunca se desecharon patrones de ocurrencia igual o mayor que 3 . Las redundancias se redujeron al $90 \%$. Se limitaron las coincidencias entre patrones reales y aleatorizados cinco veces. El nivel de significación fue de, 005 , de modo que el porcentaje para aceptar un intervalo crítico debido al azar fue del ,5\%. En ausencia de t-patterns se obtuvieron los multieventos de cada jugador. El multievento describe las múltiples conductas que pueden suceder en un contexto natural (Anguera y Blanco, 2003). En este estudio, hizo referencia al conjunto de criterios que definieron la técnica de tiro antes y a la salida del balón.

\section{RESULTADOS}

No se detectó ningún t-pattern antes de la salida del balón ni a la salida debido a que los tiros se realizaron siguiendo conductas muy dispares, que no se repitieron de forma regular en el tiempo. En las tablas 5 y 6 se muestran los diferentes multieventos ejecutados por cada jugador antes de la salida y a la salida del balón, respectivamente. Ninguno de los participantes ejecutó únicamente un tipo de multievento ni siguió las recomendaciones de la literatura atendiendo al multievento más repetido. En general, se detectó un mayor número de multieventos diferentes a la salida del balón $(n=77)$ con respecto a antes de la salida $(n=50)$.

En relación con el momento previo a la salida del balón, el jugador número 2 mostró el mayor número de multieventos diferentes $(n=6)$ y los jugadores 1 , 11 y 12 fueron los que menos multieventos diferentes mostraron $(n=3)$. Atendiendo al multievento más repetido de cada participante destaca lo siguiente. Como aspectos positivos, la mayoría de los jugadores situaron la mano de tiro debajo del balón, excepto los jugadores 4 y 10, quienes utilizaron ambas manos situadas en el lateral del balón para tirar. Todos los jugadores situaron los dos pies orientados hacia canasta y a la misma distancia de la canasta o con el pie ejecutor adelantado, excepto el jugador 3, que no situó ambos pies orientados hacia la canasta. Igualmente, la mayoría de los jugadores realizaron una flexión media del codo correspondiente a la mano de tiro, excepto los jugadores 3,8 y 9 , que lo situaron muy flexionado. Por el contrario, como aspectos negativos, siete jugadores (números $1,4,5$,
$6,8,11$ y 12) ejecutaron el tiro con salto. Además, todos los jugadores excepto el número 11 mostraron un estilo de tiro bajo.

Tabla 5

Multieventos ejecutados por los jugadores antes de la salida del balón

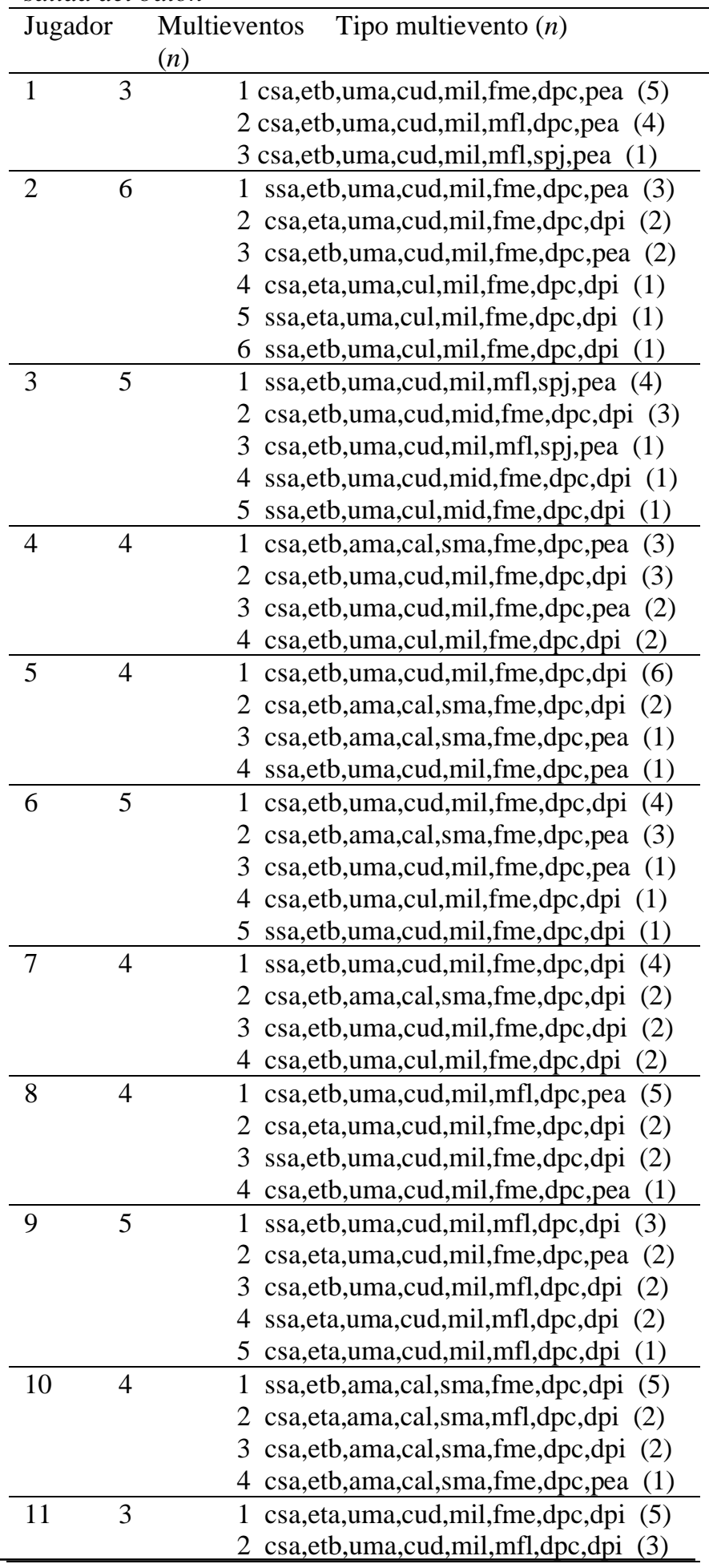




\section{Técnica de tiro libre en baloncesto formativo}

\begin{tabular}{lll}
\hline & 3 csa,etb,uma,cud,mil,fme,dpc,dpi (2) \\
\hline 12 & 1 csa,etb,uma,cud,mil,fme,dpc,dpi (7) \\
& 2 csa,etb,uma,cud,mil,mfl,dpc,dpi (2) \\
& 3 csa,eta,uma,cud,mil,fme,dpc,dpi (1) \\
\hline
\end{tabular}

Con respecto a la salida del balón, el jugador número 9 mostró el mayor número de multieventos diferentes $(n=9)$ y los jugadores 3,8 y 10 fueron los que menos multieventos diferentes mostraron $(n=4)$. Atendiendo al multievento más repetido de cada participante destaca lo siguiente. Como aspectos positivos, todos los jugadores tiraron manteniendo la mano de apoyo estática lateral, excepto los jugadores 4 y 10, que no utilizaron mano de apoyo. Igualmente, todos los jugadores orientaron el brazo de tiro hacia la canasta, excepto el jugador número 9 , que lo orientó hacia el interior. La mayoría de jugadores extendieron completamente el codo del brazo de tiro, excepto los jugadores 1,3 y 9 . Todos los jugadores realizaron una flexión completa de muñeca, excepto el jugador número 4. Por el contrario, como aspectos negativos, la mayoría de los jugadores (números 1,5 , $8,9,10,11$ y 12) ejecutaron un tiro caracterizado porque el balón abandonó la mano antes del punto de máxima altura. Sólo dos jugadores (números 8 y 9) terminaron con el brazo muy próximo a la vertical. Igualmente, sólo tres jugadores realizaron el tiro sin desplazamiento hacia delante o detrás y rotación del cuerpo (números 3,7 y 9).

Tabla 6

Multieventos ejecutados por los jugadores a la salida del balón

\begin{tabular}{|c|c|}
\hline $\begin{array}{l}\text { Juga } \\
\text { dor }\end{array}$ & Tipo multievento $(n)$ \\
\hline 1 & $\begin{array}{l}1 \text { amh,ela,elo,hca,pex,bpp,fco,dma,sro (2) } \\
2 \text { pmh,ela,elo,hin,tex,bpp,fic,dga,roi (2) } \\
3 \text { amh,ela,dlo,hin,tex,bpp,fic,dga,roi (1) } \\
4 \text { amh,ela,elo,hca,pex,bpp,fco,dmd,sro (1) } \\
5 \text { amh,ela,elo,hca,tex,bpp,fco,dga,sro (1) } \\
6 \text { amh,ela,elo,hca,tex,bpp,fco,dma,sro (1) } \\
7 \text { pmh,ela,elo,hca,tex,bpp,fco,dga,roi (1) } \\
8 \text { pmh,ela,elo,hin,tex,bpp,fco,dga,roi (1) }\end{array}$ \\
\hline 2 & $\begin{array}{l}1 \text { pmh,ela,elo,hca,tex,bpr,fco,sde,roi (3) } \\
2 \text { pmh,hab,elo,hca,tex,bpr,fco,dmd,rod (2) } \\
3 \text { amh,hab,dlo,hca,tex,bpr,fco,dmd,roi (1) } \\
4 \text { amh,hab,elo,hca,tex,bpr,fco,sde,sro (1) } \\
5 \text { pmh,ela,elo,hca,tex,bpr,fco,dmd,roi (1) } \\
6 \text { pmh,ela,elo,hca,tex,bpr,fco,dmd,sro (1) } \\
7 \text { pmh,hab,elo,hca,tex,bpr,fco,sde,roi (1) }\end{array}$ \\
\hline 3 & $\begin{array}{l}1 \text { pmh,ela,elo,hca,pex,bpr,fco,sde,sro (4) } \\
2 \text { amh,ela,dlo,hca,tex,bpr,fco,dmd,roi (3) }\end{array}$ \\
\hline
\end{tabular}

\begin{tabular}{|c|c|c|}
\hline & & $\begin{array}{l}3 \text { amh,hab,dlo,hca,tex,bpr,fco,sde,sro (2) } \\
4 \text { pmh,ela,elo,hca,pex,bpr,fco,dmd,sro (1) }\end{array}$ \\
\hline 4 & 7 & $\begin{array}{l}1 \text { pmh,nma,elo,hca,tex,bpp,fic,dgd,roi (3) } \\
2 \text { amh,ela,dlo,hca,tex,bpr,fco,dmd,roi (2) } \\
3 \text { amh,ela,dlo,hca,pex,bmp,fco,dmd,sro (1) } \\
4 \text { amh,ela,dlo,hca,pex,bpr,fco,dmd,sro (1) } \\
5 \text { amh,ela,elo,hca,pex,bpr,fco,dmd,sro (1) } \\
6 \text { pmh,ela,elo,hca,pex,bpp,fco,dmd,sro (1) } \\
7 \text { pmh,ela,elo,hca,pex,bpr,fco,dmd,sro (1) }\end{array}$ \\
\hline 5 & 7 & $\begin{array}{l}1 \text { amh,ela,elo,hca,tex,bpr,fco,dmd,roi (3) } \\
2 \text { amh,nma,elo,hca,tex,bpr,fco,dgd,sro (2) } \\
3 \text { amh,ela,elo,hca,tex,bpr,fco,dgd,sro (1) } \\
4 \text { amh,nma,dlo,hca,tex,bpr,fco,dgd,sro (1) } \\
5 \text { pmh,ela,elo,hca,tex,bpr,fco,dmd,sro (1) } \\
6 \text { pmh,ela,elo,hca,tex,bpr,fco,sde,sro (1) } \\
7 \text { pmh,ela,elo,hin,tex,bpr,fco,dmd,roi (1 }\end{array}$ \\
\hline 6 & 8 & $\begin{array}{l}1 \text { pmh,ela,elo,hca,tex,bpr,fco,dmd,roi (2) } \\
2 \text { pmh,nma,elo,hca,tex,bpp,fic,dgd,roi (2) } \\
3 \text { amh,ela,elo,hca,tex,bpr,fco,dgd,sro (1) } \\
4 \text { amh,nma,elo,hca,tex,bpr,fco,dgd,sro (1) } \\
5 \text { pmh,ela,elo,hca,tex,bpp,fco,dmd,roi (1) } \\
6 \text { pmh,ela,elo,hca,tex,bpp,fco,dmd,sro (1) } \\
7 \text { pmh,ela,elo,hca,tex,bpp,fco,sde,roi (1) } \\
8 \text { pmh,ela,elo,hca,tex,bpr,fco,dgd,sro (1) } \\
\end{array}$ \\
\hline 7 & 7 & $\begin{array}{l}1 \text { pmh,ela,elo,hca,tex,bpr,fco,sde,sro (3) } \\
2 \text { pmh,ela,elo,hca,tex,bpr,fco,dmd,roi (2) } \\
3 \text { amh,ela,elo,hca,tex,bpr,fco,dmd,roi (1) } \\
4 \text { amh,nma,dlo,hca,tex,bpr,fco,dgd,sro (1) } \\
5 \text { amh,nma,elo,hca,tex,bpr,fco,dgd,sro (1) } \\
6 \text { pmh,ela,elo,hca,tex,bpp,fco,sde,sro (1) } \\
7 \text { pmh,ela,elo,hca,tex,bpr,fco,dmd,sro (1) }\end{array}$ \\
\hline 8 & 4 & $\begin{array}{l}1 \text { amh,ela,elo,hca,tex,bmp,fco,dma,rod (3) } \\
2 \text { amh,hab,elo,hca,tex,bpr,fco,dmd,sro (3) } \\
3 \text { pmh,ela,elo,hca,tex,bpp,fco,sde,sro (2) } \\
4 \text { pmh,hab,elo,hca,tex,bpr,fco,dmd,sro (2) }\end{array}$ \\
\hline 9 & 9 & $\begin{array}{l}1 \text { amh,ela,elo,hin,pex,bmp,fco,sde,sro (2) } \\
2 \text { amh,ela,elo,hca,pex,bmp,fco,dmd,sro (1) } \\
3 \text { amh,ela,elo,hca,tex,bmp,fco,dga,rod (1) } \\
4 \text { amh,ela,elo,hca,tex,bmp,fco,dma,rod (1) } \\
5 \text { amh,ela,elo,hca,tex,bpr,fco,sde,sro (1) } \\
6 \text { amh,ela,elo,hin,pex,bmp,fco,dmd,sro (1) } \\
7 \text { amh,hab,elo,hca,tex,bpr,fco,dmd,sro (1) } \\
8 \text { amh,hab,elo,hca,tex,bpr,fco,sde,sro (1) } \\
9 \text { pmh,ela,elo,hca,pex,bmp,fco,sde,sro (1) }\end{array}$ \\
\hline 10 & 4 & $\begin{array}{l}1 \text { amh,nma,elo,hca,tex,bpr,fco,dmd,sro (4) } \\
2 \text { amh,nma,elo,hca,tex,bpr,fco,sde,sro (3) } \\
3 \text { amh,nma,elo,hca,tex,bpr,fco,sde,sri (2) } \\
4 \text { pmh,nma,elo,hca,tex,bpr,fco,dmd,sro (1) }\end{array}$ \\
\hline 11 & 6 & $\begin{array}{l}1 \text { amh,ela,elo,hca,tex,bpr,fco,dmd,sro (4) } \\
2 \text { amh,ela,elo,hin,pex,bpr,fco,dmd,sro (2) } \\
3 \text { amh,ela,elo,hca,tex,bmp,fco,dmd,sro (1) } \\
4 \text { amh,ela,elo,hca,tex,bpr,fic,dmd,sro (1) } \\
5 \text { pmh,ela,elo,hca,tex,bmp,fco,dmd,sro (1) } \\
6 \text { pmh,ela,elo,hin,pex,bpr,fco,dmd,sro (1) }\end{array}$ \\
\hline 12 & 6 & 1 amh,ela,elo,hca,tex,bpr,fco,dmd,sro (3) \\
\hline
\end{tabular}




\section{Díaz-Aroca, \& Arias-Estero}

\author{
2 amh,ela,elo,hca,tex,bmp,fco,dmd,sro (2) \\ 3 amh,ela,elo,hin,pex,bpr,fco,dmd,sro (2) \\ 4 amh,ela,elo,hca,tex,bpr,fco,dmd,rod (1) \\ 5 pmh,ela,elo,hca,tex,bmp,fco,dmd,sro (1) \\ 6 pmh,ela,elo,hca,tex,bpr,fco,dmd,sro (1)
}

\section{DISCUSIÓN}

El objetivo del presente trabajo fue determinar si la técnica de tiro libre ejecutada por jugadores de baloncesto de entre 9 y 11 años no participantes en ninguna selección coincidió con la técnica reportada por la literatura y estudios previos. Los resultados confirmaron la hipótesis, tanto antes de la salida del balón como a la salida del mismo. La técnica de tiro libre ejecutada por los jugadores analizados fue diferente a la técnica ideal propuesta por la literatura y a la ejecutada por los jugadores participantes en selecciones de estudios previos (ASEP, 1996; Del Rio, 2002; Garzón et al., 2011, 2014; Krause y Nelson, 2019; Montero, 2013).

A diferencia de los resultados obtenidos por Garzón et al. $(2011,2014)$, en el presente trabajo no se detectaron t-patterns. En la misma línea y en contraste con los resultados del estudio de Garzón et al. (2014), ningún jugador del presente trabajo realizó un tiro con una ejecución técnica que cumpliera completamente con el modelo teórico descrito por la literatura (ASEP, 1996; Del Rio, 2002; Krause y Nelson, 2019; Montero, 2013). Estas inconsistencias entre estudios pudieron deberse a las diferencias entre los participantes. Es ampliamente aceptado que los jugadores elegidos para participar con sus respectivas selecciones presentan unas características físicas más desarrolladas que los que no son seleccionados (Arrieta, Torres-Unda, Gil, y Irazusta, 2015; TorresUnda et al., 2013). Igualmente, los jugadores seleccionados disfrutan de más horas de entrenamiento y por tanto adquieren más experiencias que sus iguales que no pueden acceder a dicha formación (Werneck et al., 2016).

Antes de la salida del balón se observó el criterio tiro con salto en el multievento más repetido por siete de los 12 jugadores (números 1, 4, 5, 6, 8, 11 y 12). Esto ocurrió a pesar de que un tiro sin salto es más apropiado para encestar, gracias a que la estabilidad durante el tiro es mayor (Wissel, 2012). Consecuentemente, la flexión del codo fue excesiva en el caso de los jugadores 3,8 y 9, con el fin de aportar más propulsión al balón antes de su salida. En la misma línea, dos jugadores (4 y 10) ejecutaron el tiro impulsando el balón con ambas manos situadas en el lateral. La ausencia de mano de apoyo dificultó la estabilidad del tiro y su precisión (Zuzik, 2011). Además, todos los participantes ejecutaron la mayor proporción de sus tiros con estilo bajo. Sin embargo, el estilo alto favorece el éxito del tiro al elevar la altura de liberación del balón y posibilitar la visualización de la canasta durante toda la ejecución (Arias, 2012b).

Con respecto a la salida del balón, ocho de los 12 jugadores ejecutaron un tiro donde el balón abandonó la mano antes del punto de máxima altura (números $1,5,8,9,10,11$ y 12). Este criterio contrastó con las afirmaciones de la literatura ya que existe una relación directa entre la altura de salida del balón y conseguir encestar (Khlifa, et al., 2012; Oudejans, Karamat, y Stolk, 2012). De manera similar, 10 jugadores dispusieron el brazo más próximo a la horizontal que a la vertical, limitando la trayectoria parabólica del balón, probablemente para ejercer un movimiento de empuje sobre el mismo. Este es un recurso habitualmente realizado por los niños para conseguir que el balón alcance el aro (Rojas, Sánchez, Cepero, Soto, y Gutiérrez, 2000). No obstante, esta conducta limita las posibilidades de encestar al disminuir el ángulo y la altura de salida del balón y aumentar la velocidad (Miller y Bartlett, 1996; Tran y Silverberg, 2008). A medida que aumenta el ángulo de liberación del balón también aumenta el arco de su trayectoria. Una trayectoria más parabólica favorece el componente vertical de la velocidad del balón frente a la horizontal. Como consecuencia, el tiro tiende a ser más preciso. Si por el contrario el balón golpease en el aro o el tablero, rebotaría hacia arriba, en vez de horizontalmente, aumentando las opciones de encestar (Schneider y Williams, 2010). Por otro lado, sólo tres jugadores realizaron el tiro sin desplazamiento y sin rotación del cuerpo (números 3, 7 y 9). Sin embargo, estos desplazamientos inciden negativamente sobre la precisión del tiro al generar un mayor desequilibrio en los jugadores (Miller y Bartlett, 1996; Okazaki, Lamas, Okazaki, y Rodacki, 2013).

En consecuencia con las ideas abordadas en los párrafos previos, tanto antes de la salida del balón 


\section{Técnica de tiro libre en baloncesto formativo}

como a la salida, ninguno de los jugadores ejecutó un mismo multievento en todos sus tiros libres ni hubo estabilidad temporal en los mismos. Probablemente, las conductas mostradas por los participantes ocurrieron porque muchos jugadores a estas edades no presentan la fuerza que demandan el peso del balón, la distancia a la canasta y la altura de la misma para alcanzar el aro y como consecuencia encestar (Struzik, Rokita, Pietraszewski, y Popowczak, 2014). Además, se observó que la variabilidad de los multieventos fue mayor a la salida del balón que antes de la salida. Cuando las condiciones en las que se ejecuta el tiro libre demandan una fuerza de la que carecen los niños, inicialmente ellos tienen que realizar ajustes en sus segmentos corporales normalmente antes de la salida del balón (Mullineaux y Uhl, 2010). Posteriormente, motivado por su nivel de fuerza y las adaptaciones realizadas en sus segmentos corporales antes de la salida del balón, el número de movimientos compensatorios aumenta y como consecuencia se incrementa la variabilidad de las conductas a la salida (Mullineaux y Uhl, 2010; Pojskić, Šeparović, Muratović y Užičanin, 2014). Es por ello que 9 de los 12 jugadores (números 1, 2, 4, 5, $6,7,9,11$ y 12) utilizaron diferentes estrategias de ajuste en su técnica de tiro en más de la mitad de los lanzamientos a la salida del balón. Es decir, el 60\% de los tiros de 9 jugadores fueron ejecutados utilizando diferentes tipos de multieventos. Además, si la técnica no es entrenada de manera específica, el patrón de tiro resulta altamente inestable (Miller y Bartlett, 1996). Especialmente llamativo fue el caso del jugador número 2, quien mostró seis tipos diferentes de multieventos antes de la salida del balón. Igualmente, el jugador número 9 mostró nueve tipos diferentes de multieventos a la salida del balón. Esto es, prácticamente en todos sus tiros libres varió algún criterio de la técnica.

\section{CONCLUSIONES}

En conclusión, los jugadores del presente estudio, quienes no compitieron con la selección autonómica, mostraron una técnica de tiro libre diferente a la de jugadores de la misma edad de estudios previos que competían con su selección. A su vez, la técnica fue alejada de la gestoforma correcta recomendada por la literatura. A pesar de las diferencias con la literatura, estos resultados fueron esperados dado que la gran mayoría de los jugadores de menos de 12 años suelen estar limitados por sus características físicas y técnicas (Ibáñez, Mazo, Nascimento, y García-Rubio, 2018) en relación con las condiciones de práctica (en este caso el peso del balón, la distancia a la canasta y la altura de la misma). Lo anterior no significa que la técnica de tiro libre determine únicamente estar o no en una selección. Por el contrario, los resultados llevan a plantearse si las reglas de juego están adaptadas a la mayoría de jugadores de minibasket debido a sus características cineantropométricas. La falta de adecuación de las reglas de juego a estas edades ya fue puesto de manifiesto en estudios anteriores (Arias, 2012a, b). Sin embargo, si existe una acción de juego que deba trabajarse estrictamente de manera técnica en baloncesto de formación ese es el tiro libre, al ser una acción cerrada directamente relacionada con el éxito del juego. Es importante conocer qué criterios del tiro libre son susceptibles de ser entrenados y mejorados, puesto que mejorar la técnica en etapas de formación permite aumentar el éxito en el tiro tanto a corto plazo, durante la infancia, como a largo plazo, al evitar la adquisición de conductas complejas de cambiar en la etapa adulta.

\section{APLICACIONES PRÁCTICAS}

En el presente trabajo se utilizó un instrumento de observación ad hoc fácilmente aplicable por los entrenadores. El instrumento permite la obtención de información práctica y directamente aplicable a la realidad para la evaluación y trabajo del tiro libre de jugadores de baloncesto de menos de 12 años. Este les ayudará a analizar la técnica diferenciando lo que ocurre antes de la salida del balón y a la salida del mismo. A través del mismo, se comprobó que los jugadores analizados, los cuales no jugaban en ninguna selección, ejecutaron una técnica de tiro que contrastaba en varios criterios con las recomendaciones propuestas por la literatura. En el presente trabajo estas limitaciones implicaron la ejecución: (a) con salto, (b) estilo bajo, (c) brazo de tiro muy flexionado, (d) salida del balón antes del punto de máxima altura, (e) brazo de tiro más horizontal que vertical a la salida del balón y (f) con desplazamiento y rotación del cuerpo. Se recomienda a los entrenadores que incluyan tareas en sus entrenamientos para trabajar el tiro libre y en concreto para mejorar los errores mencionados. Si las condiciones de práctica no posibilitan la ejecución 


\section{Díaz-Aroca, \& Arias-Estero}

del tiro con la técnica ideal, dichas condiciones deben ser adaptadas a las características de los niños.

\section{REFERENCIAS}

1. Anguera, M. T., y Blanco, A. (2003). Registro y codificación en el comportamiento deportivo. En A. Hernández (Coord.), Psicología del deporte (Vol. 2). Metodología (p. 6-34). Buenos Aires: Efdeportes (www.efdeportes.com).

2. Anguera, M. T., Blanco-Villaseñor, A., Hernández-Mendo, A., y Losada, J. L. (2011). Observational designs: Their suitability and application in sports psychology. Cuadernos de Psicología del Deporte, 11, 3-76.

3. Anguera, M. T., y Hernández-Mendo, A. (2014). Técnicas de análisis en estudios observacionales en ciencias del deporte. Cuadernos de Psicología del Deporte, 15, 13-30. doi:10.4321/s157884232015000100002

4. Arias, J. L. (2012a). Influence of ball weight on shot accuracy and efficacy among 9-11-year-old male basketball players. Kinesiology, 44, 52-59.

5. Arias, J. L. (2012b). Performance as a function of shooting style in basketball players under 11 years of age. Perceptual and Motor Skills, 114(2), $\quad$ 446-456. doi:10.2466/05.11.pms.114.2.446-456

6. Arrieta, H., Torres-Unda, J., Gil, S. M., y Irazusta, J. (2015). Relative age effect and performance in the U16, U18 and U20 European Basketball Championships. Journal of Sports Sciences, $\quad 34, \quad 1530-1534$. doi:10.1080/02640414.2015.1122204

7. ASEP. (1996). Coaching youth basketball (2nd edition). Champaign, Il: Human Kinetics.

8. Blanco-Villaseñor, A., Castellano, J., HernándezMendo, A., Sánchez-López, C. R., y Usabiaga, O. (2014). Aplicación de la TG en el deporte para el estudio de la fiabilidad, validez y estimación de la muestra. Revista de Psicología del Deporte, 23, 131-137.

9. Chase, M. A., Ewing, M. E., Lirgg, C. D., y George, T. R. (1994). The effects of equipment modification on children's self-efficacy and basketball shooting performance. Research
Quarterly for Exercise and Sport, 65, 159-168. doi:10.1080/02701367.1994.10607611

10. Cohen, J. (1968). Weighted kappa: Nominal scale agreement with provision for scaled disagreement of partial credit. Psychological Bulletin, 70, 213-220. doi:10.1037/h0026256

11. Da Silva, L., Pereira-Monfredini, C., y Teixeira, L. (2017). Improved children's motor learning of the basketball free shooting pattern by associating subjective error estimation and extrinsic feedback. Journal of Sports Sciences, 35 , 1825-1830. doi:10.1080/02640414.2016.1239025

12. De Oliveira, R. F., Oudejans, R. R. D., y Beek, P. J. (2006). Late information pick-up is preferred in basketball jump shooting. Journal of Sports Sciences, 24, 933-940. doi:10.1080/02640410500357101

13. Del Río, J. A. (2002). Metodología del baloncesto. Barcelona, España: Paidotribo.

14. Diana, B., Zurloni, V., Elia, M., Cavalera, C. M., Jonsson, G. K., y Anguera, M. T. (2017) How game location affects soccer performance: $\mathrm{T}$ pattern analysis of attack actions in home and away matches. Frontiers in Psychology, 8, 1415. doi:10.3389/fpsyg.2017.01415

15. Garzón, B., Lapresa, D., Anguera, M. T., y Arana, J. (2011). Análisis observacional del lanzamiento de tiro libre en jugadores de baloncesto base. Psicothema, 23, 851-857.

16. Garzón, B., Lapresa, D., Anguera, M. T., y Arana, J. (2014). Del minibasket al baloncesto: Efectos de la actual configuración reglamentaria en el patrón técnico de lanzamiento de tiro libre. Revista de Psicología del Deporte, 23, 77-85. doi:10.24310/riccafd.2014.v3i1.6180

17. Hernández-Mendo, A., Blanco-Villaseñor, A., Pastrana, J. L., Morales-Sánchez, V., RamosPérez, F. J. (2016). SAGT: Aplicación informática para análisis de generalizabilidad. Revista Iberoamericana de Psicología del Ejercicio y el Deporte, 11, 77-89.

18. Ibáñez, S. J., Mazo, A., Nascimento, J., y GarcíaRubio, J. (2018). The relative age effect in under18 basketball: Effects on performance according 


\section{Técnica de tiro libre en baloncesto formativo}

to playing position. PLOS ONE, 13, e0200408. doi:10.1371/journal.pone.0200408

19. Isaacs, L. D., y Karpman, M. B. (1981). Factors effecting children's basketball shooting performance: A log-linear analysis. Carnegie School of Physical Education and Human Movement, 1, 29-32.

20. Khlifa, R., Aouadi, R., Hermassi, S., Chelly, M. S., Jlid, C., y Gabbett, T. J. (2012). Kinematic adjustments in the basketball free throw performed with a reduced hoop diameter rim. International Journal of Sports Science \& Coaching, 2, 371-381. doi:10.1260/17479541.7.2.371

21. Klostermann, A., Panchuk, D., y Farrow, D. (2017). Perception-action coupling in complex game play: Exploring the quiet eye in contested basketball jump shots. Journal of Sports Sciences, 36, 1054-1060. doi:10.1080/02640414.2017.1355063

22. Krause, J. V., y Nelson, C. (2019). Baloncesto destrezas y ejercicios: La guía superventas sobre los fundamentos del baloncesto. Madrid: Tutor Ediciones.

23. Lapresa, D., García, J., Arana, J., y Garzón, B. (2011). Análisis de patrones temporales en las rutinas gestuales previas al tiro libre de baloncesto, en la categoría alevín. Revista de Psicología del Deporte, 20(2), 383-400. doi:10.24310/riccafd.2014.v3i1.6180

24. Li, Y., y Feng, T. (2020) The effects of sport expertise and shot results on basketball players' action anticipation. PLOS ONE, 15, e0227521. doi:10.1371/journal.pone.0227521

25. Lorenzo, J., Lorenzo, A., Conte, D., y Giménez, M. (2019). Long-term analysis of elite basketball players' game-related statistics throughout their careers. Frontiers in Psychology, 10, 421. doi:10.3389/fpsyg.2019.00421

26. Magnusson, M. S. (1996) Hidden real-time patterns in intra- and inter-individual behavior. European Journal of Psychological Assessment, 12, 112-123. doi:10.1027/1015-5759.12.2.112

27. Magnusson, M. S. (2000). Discovering hidden time patterns in behavior: T-patterns and their detection. Behavior Research Methods,
Instruments, \& Computers, 32, 93-110. doi:10.3758/bf03200792

28. Magnusson, M. S., Burgoon, J. K., y Casarrubea, M. (2016). Discovering hidden temporal patterns in behavior and interaction. New York, NY: Springer-Verlag.

29. Miller, S., y Bartlett, R. (1996). The relationship between basketball shooting kinematics, distance and playing position. Journal of Sports Sciences, 14, 243-253. doi:10.1080/02640419608727708

30. Montero, A. (2013). Didáctica del baloncesto. Barcelona, España: Paidotribo.

31. Morgulev, E., Azar, O., y Bar-Eli, M. (2019). Searching for momentum in NBA triplets of free throws. Journal of Sports Sciences, 38, 390-398. doi:10.1080/02640414.2019.1702776

32. Mullineaux, D., y Uhl, T. (2010). Coordinationvariability and kinematics of misses versus swishes of basketball free throws. Journal of Sports Sciences, 28, 1017-1024. doi:10.1080/02640414.2010.487872

33. Okazaki, V., Lamas, L., Okazaki, F., y Rodacki, A. (2013). The effect of distance increase on basketball shot performed by children. Motricidade, 9, 61-72.

34. Okubo, H., y Hubbard, M. (2016). Comparison of shooting arm motions in basketball. Procedia Engineering, $\quad 147, \quad 133-138$. doi:10.1016/j.proeng.2016.06.202

35. Oudejans, R., Karamat, R., y Stolk, H. (2012). Effects of actions preceding the jump shot on gaze behavior and shooting performance in elite female basketball players. International Journal of Sports Science and Coaching, 7, 255-267. doi: 10.1260/1747-9541.7.2.255

36. Perreault, M., y French, K. (2015). Externalfocus feedback benefits free-throw learning in children. Research Quarterly for Exercise and Sport, 86, 422-427. doi:10.1080/02701367.2015.1051613

37. Pojskic, H., Šeparović, V., Muratović, M., y Užičanin, E. (2014). The relationship between physical fitness and shooting accuracy of professional basketball players. Motriz, 20, 408417. doi:10.1590/s1980-65742014000400007 


\section{Díaz-Aroca, \& Arias-Estero}

38. Prieto-Lage, I., Artigues-Ribas, L., y GutiérrezSantiago, A. (2020). Patrones técnico-tácticos del lanzador y el portero en los penales de la liga española de fútbol durante la temporada 2016-17 mediante t-patterns y coordenadas polares. Cuadernos de Psicología del Deporte, 20, 166180.

39. Prieto-Lage., I, Rodríguez-Souto, M., Prieto, M. A, Gutiérrez-Santiago, A. (2020). Technical analysis in Tsuri-goshi through three complementary observational analysis. Physiology \& Behavior, 216, 112804. doi:10.1016/j.physbeh.2020.112804

40. Regimbal, C., Deller, J., y Plimpton, C. (1992). Basketball size as related to children's preference, rated skill and scoring. Perceptual and Motor Skills, 75(3), 867-872. doi:10.2466/pms.1992.75.3.867

41. Rojas, F. J., Sánchez, A., Cepero, M., Soto, V. M., y Gutiérrez, M. (2000). Diferencias biomecánicas entre jugadores escolares y de alto rendimiento en el lanzamiento en salto en baloncesto. Biomecánica, 8(1), 3-14. doi:10.20868/upm.thesis.52660

42. Satern, M., Messier, P., y Keller-McNulty, S. (1989). The effect of ball size and basket height on the mechanics of the basketball free throw. Journal of Human Movement Studies, 16, 123137.

43. Schneider, R., y Williams, C. (2010). Success rates of underhand and overhand free-throws as novel skills. Journal of Physical Education and Sport, 27(2), 13-20.

44. Struzik, A., Rokita, A., Pietraszewski, B., y Popowczak, M. (2014).Accuracy of replicating static torque and its effect on shooting accuracy in young basketball players. Human Movement, 15, 216-220. doi:10.1515/humo-2015-0014

45. Torres-Unda, J., Zarrazquin, I., Gravina, L., Zubero, J., Seco, J., Gil, S. M., ...Irazusta J. (2016). Basketball performance is related to maturity and relative age in elite adolescent players. Journal of Strength and Conditioning Research, 30, 1325-1332. doi:10.1519/JSC.0000000000001224

46. Tran, C., y Silverberg, L. (2008). Optimal release conditions for the free throw in men's basketball.
Journal of Sports Sciences, 26, 1147-1155. doi:10.1080/02640410802004948

47. Werneck, F. Z., Coelho, E. F., de Oliveira, H. Z., Ribeiro Júnior, D. B., Almas, S. P., de Lima, J. R. P., ...Figueiredo, A. J. (2016). Relative age effect in Olympic basketball athletes. Science \& Sports, $\quad 31$ 158-161. doi:10.1016/j.scispo.2015.08.004

48. Wissel, H. (2012). Basketball: Steps to success (3rd edition). Champaign, Il: Human Kinetics.

49. Zuzik, P. (2011). Free throw shooting effectiveness in basketball matches of men and women. Sport Science Review, 20, 149-160. doi:10.2478/v10237-011-0059-y 